\title{
PRODUÇÃO CIENTÍFICA E A TEORIA FUNDAMENTADA NOS DADOS NA PRÁTICA DE ENFERMAGEM COM CRIANÇAS NA ATENÇÃO PRIMÁRIA EM SAÚDE
}

\author{
SCIENTIFIC PRODUCTION AND A GROUNDED THEORY IN \\ NURSING PRACTICE WITH CHILDREN IN PRIMARY HEALTH CARE
}

Larissa Soares Mariz ${ }^{1}$

Joselane Izaquiel Marinho

\begin{abstract}
RESUMO: A Atenção Primária de Saúde é o primeiro nível de contato da comunidade com a assistência de saúde. No cuidado a criança esse nível de assistência envolve todos os aspectos e momentos de crescimento e desenvolvimento saudável. A Teoria Fundamentada nos Dados como método científico, fornece base para a prática qualificada de enfermagem na atenção às crianças. OBJETIVO: integrar estudos que abordem o cuidado de enfermagem às crianças na APS e que aplicaram o método de TFD em suas pesquisas. MÉTODO: revisão integrativa realizada entre Dezembro de 2014 e Janeiro de 2015 com artigos em português, inglês ou espanhol abordando a prática de enfermagem na APS com crianças e utilizando a metodologia da TFD e publicados nos últimos cinco anos. Foram excluídos: editoriais; cartas ao editor; artigos que abordassem atenção às crianças fora da APS ou por outros cuidadores que não a enfermagem; e artigos de revisão de literatura sistemática, integrativa ou metodológica. A revisão foi composta por 9 artigos analisados. RESULTADOS: Percebeu-se que a Teoria Fundamentada nos Dados tem sido utilizada em pesquisas da enfermagem e buscam perceber a interação da família das crianças como receptoras do cuidado. Metodologicamente os artigos demonstram estar bem estruturados e produzem modelos teóricos com aplicabilidade nas práticas do profissionais de enfermagem e úteis para a Atenção Básica. CONCLUSÃO: A metodologia em questão é um instrumento utilizado nas pesquisas de enfermagem e produzem conhecimento efetivo para a pratica do cuidado de crianças na Atenção primária de saúde.
\end{abstract}

Palavras chave: Atenção Básica. Cuidado da Criança. Enfermagem. Teoria de Enfermagem. Teoria Fundamentada nos Dados.

\footnotetext{
${ }^{1}$ Professora Doutora do Curso Bacharelado em Enfermagem da Universidade Federal de Campina Grande - Paraíba. E-mail: larissamariz@gmail.com.

${ }^{2}$ Graduanda do curso de bacharelado em EnfermagemdaUniversidade Federal de Campina Grande.
} 
ABSTRACT: Primary Health Care is the community's first level of contact with health care. In child care, this level of assistance involves all aspects and moments of healthy growth and development. Grounded Theory as a scientific method, provides a basis for qualified nursing practice in childcare. It aimed to integrate studies that address nursing care for children in PHC and that applied the PDT method in their research. Method: an integrative review carried out between December 2014 and January 2015 with articles in Portuguese, English or Spanish addressing the practice of nursing in PHC with children and using the TFD methodology and published in the last five years. The following were excluded: editorials; letters to the editor; articles that addressed care for children outside PHC or by caregivers other than nursing; and systematic, integrative or methodological literature review articles. The review consisted of 9 articles analyzed. As a result, it was noticed that Grounded Theory has been used in nursing research and seeks to perceive the interaction of the children's family as recipients of care. Methodologically, the articles show to be well structured and produce theoretical models with applicability in the practices of nursing professionals and useful for Primary Care. It is concluded that the methodology in question is an instrument used in nursing research and produces effective knowledge for the practice of child care in Primary Health Care.

Keywords: Primary Health Care. Child Care. Nursing. Nursing Theory. Grounded Theory. 


\section{INTRODUÇÃO}

De acordo com Declaração de Alma Ata a definição de Atenção Primária em Saúde (APS) revela uma atenção essencial em saúde com base em métodos práticos, científicos e socialmente aceitáveis, com tecnologia disponibilizada universalmente a indivíduos e famílias na comunidade por meio de sua plena participação e a um custo que a comunidade e o país podem manter. Preferencialmente, a APS é o primeiro nível de contato dos indivíduos, a família e a comunidade com os serviços de saúde, assim constitui o primeiro elemento de um processo contínuo de atenção em saúde (WHO, 1978).

A partir da adoção das práticas de APS, alcançou-se um importante progresso no desenvolvimento humano e de saúde na Região das Américas. Dentre elas estão a melhoria de grande parte dos valores médios de quase todos os indicadores de saúde em boa parte dos países na Região (PAHO, 2007).

No que se refere às ações da APS à criança, são direcionadas a organizar os serviços de forma a impulsionar as diversas formas de cuidar e alcançar as muitas circunstâncias que envolvem a vida da criança (SOUSA, 2010). Para alcançar esse cuidado faz-se necessário excluir a forma de agir isolada de um único profissional, a fim de que não se perca o olhar ampliado para as necessidades do crescimento e desenvolvimento infantil, incluindo a família e o ambiente no qual a criança está inserida (SOUSA, 2010).

A enfermagem se associa a esse sistema, por ser uma profissão que agrega ciência, arte e sensibilidade (GASTALDON, 2007). Contudo, para adentrar o cotidiano das crianças e suas famílias, requer dos profissionais de enfermagem da APS um olhar qualificado para a identificação dos problemas e exige a utilização de um conjunto de tecnologias adequadas (SARTI, 2012). Nesse sentido, Fawcett (2004) refere que a elaboração de teorias de médio alcance contribui para o avanço da disciplina de enfermagem. 
Por isso, a utilização da Teoria Fundamentada nos Dados (TFD) para produção científica de teorias de pequeno ou médio alcance serve de base para a prática qualificada de enfermagem na atenção às crianças na APS. A TFD trata-se de um método para construção de teoria com base nos dados investigados de determinada realidade, de maneira indutiva ou dedutiva que, mediante a organização em categorias conceituais, possibilita a explicação do fenômeno investigado (DANTAS et al, 2009). Como produto da aplicação deste método, buscase estabelecer modelos teóricos ou reflexões teóricas (DANTAS et al, 2009).

Assim, considerando as diferentes áreas de pesquisa que envolve a atenção de enfermagem às crianças na APS, questionou-se: Quais os objetivos, questão de pesquisa, linha de conhecimento teórico sobre TFD, base filosófica, desenvolvimento de modelos teóricos, produtos desenvolvidos, avanços e impactos na prática, categorias, afirmações teóricas e conclusões dos estudos que utilizaram como metodologia a TFD com foco em crianças, na APS? Esta pesquisa teve por objetivo integrar estudos que abordem o cuidado de enfermagem às crianças na APS e que aplicaram o método de TFD em suas pesquisas.

\section{MÉTODO}

Como método, utilizou-se a revisão integrativa, a qual tem sido amplamente utilizada para reunir achados de estudos de diferentes metodologias, de forma a integrar e/ou sintetizar resultados sem modificar a natureza epistemológica dos estudos empíricos incluídos. (SOUZA, 2010). Nesta pesquisa, adotaram-se os seis passos para desenvolvimento do método com rigor. São eles: estabelecer hipótese/pergunta de pesquisa, selecionar a amostra, categorizar os estudos, analisar os estudos inclusos na pesquisa, interpretar os resultados e apresentar a revisão ou síntese do conhecimento (MENDES, 2008).

Assim, foram incluídos artigos disponíveis nos idiomas português, inglês ou espanhol; que abordassem a prática de enfermagem na APS com crianças e utilizaram a metodologia da TFD e publicados nos últimos cinco anos a partir da data 
de realização da busca nas bases de dados. Foram excluídos: editoriais; cartas ao editor; artigos que abordassem atenção às crianças fora da APS ou por outros cuidadores que não a enfermagem; e artigos de revisão de literatura sistemática, integrativa ou metodológica. Para critério da faixa etária, adotou-se o critério da OMS, crianças entre zero e nove anos de idade (WHO, 2012).

A busca ocorreu entre Dezembro de 2014 e Janeiro de 2015, em seis bases de dados eletrônicas: SCOPUS, CINAHL (Cumulative Index to Nursing and Allie Health Literature), PUBMED (National Libary os Medicine), LILACS (Literatura Latino-americana e do Caribe em Ciências Sociais e da Saúde), BDENF (Base de dados da Enfermagem) e SCIELO (Scientific Eletronic Library Online).

Foram utilizados os Descritores de Saúde (DECs), indexados e não indexados, seus respectivos MESHs (Medical Subject Headings) e o operador booleano AND nos seguintes cruzamentos: 1. Enfermagem AND Teoria de Enfermagem AND Cuidado da criança AND Atenção básica AND Teoria Fundamentada nos dados; 2. Atenção básica AND Cuidado da Criança AND Pesquisa qualitativa AND Teoria Fundamentada nos dados; 3. Enfermagem AND Cuidado da criança AND Saúde da criança AND Pesquisa qualitativa AND Teoria Fundamentada nos dados.

Foram identificados 6.407 estudos primários. Após leitura do título e resumo, realizou- se seleção e avaliação dos artigos de acordo com a questão norteadora e os critérios de inclusão definidos previamente. Assim, foram selecionados 39 estudos. Destes, 10 foram excluídos por se apresentarem repetidos nas bases BDENF e LILACS em relação aos contidos na SCOPUS e PUBMED. Após leitura do artigo completo e nova aplicação dos critérios de inclusão e exclusão, 2 foram excluídos por não tratar de estudo primário, 3 por abordar cuidado de enfermagem fora da APS, 8 por não incluir a faixa etária da infância, 4 pelo fato do estudo não descrever cuidados desempenhados pela enfermagem e 2 porque utilizaram outro tipo de metodologia que não a TFD.

Após seleção dos artigos para compor a amostra do estudo, procedeu-se com a análise de relevância e qualidade metodológica de acordo com formulário para avaliação de estudos qualitativos elaborados pelo Critical Appraisal Skills 
Programme (CASP). Mediante avaliação, um dos estudo alcançou índice menor que sete e foi excluído (CASP, 2013).

Finalmente o corpus da revisão foi composto por 9 artigos. O processo de análise envolveu tradução do vernáculo, leitura, releitura e preenchimento do instrumento para captação dos dados. Este foi elaborado pelos próprios autores para registro das seguintes informações e posterior análise: caracterização do estudo (título, formação do autor principal, temática abordada, ano e periódico de publicação, objetivo do estudo e questão norteadora); Informações metodológicas (população e amostra, grupos amostrais, período de coleta de dados, local do estudo, instrumentos utilizados para coleta de dados); Resultados (categorias desenvolvidas, subcategorias, desenvolvimento de modelo teórico, categoria central, produto gerado para atender ao objetivo do estudo, impacto na prática de saúde da população estudada) e conclusões (avanços para atenção à criança na APS e aplicabilidade na prática profissional).

Os dados foram transcritos em uma planilha EXCEL e analisados posteriormente buscando integrar e sintetizar os dados coletados de forma a elaborar categorias para posterior discussão. Após desenvolvimento do artigo, aplicou-se check list proposto por Soares (2014) para verificação do cumprimento das etapas de desenvolvimento da revisão integrativa com o rigor metodológico necessário.

\section{RESULTADOS}

Para presente revisão integrativa nove artigos científicos foram selecionados a fim de responder o objetivo da pesquisa. Buscando caracterizá-los, foram descritos no quadro 1 os autores, ano de publicação e título dos artigos. 
Quadro 1: Descrição dos autores, ano de publicação e títulos dos artigos selecionados.

\begin{tabular}{|c|c|c|l|}
\hline ARTIGO & AUTORES & ANO & \multicolumn{1}{|c|}{ TítULO } \\
\hline A & Dahlgren & 2012 & $\begin{array}{l}\text { Public health nurses' barriers and facilitators to the use } \\
\text { of research in consultations about childhood } \\
\text { vaccinations. }\end{array}$ \\
\hline B & Maenpaa & 2012 & $\begin{array}{l}\text { Family_school nurse partnership in primary school health } \\
\text { care. }\end{array}$ \\
\hline C & Sousa & 2012 & $\begin{array}{l}\text { Qualificando o cuidado à criança na atenção Primária de } \\
\text { Saúde. }\end{array}$ \\
\hline D & Sue & 2011 & $\begin{array}{l}\text { Caring for children dying from cancer at home: a } \\
\text { qualitative study of the experience of primary care } \\
\text { practitioners. }\end{array}$ \\
\hline E & Sousa & 2011 & $\begin{array}{l}\text { londições limitadoras para a integralidade do cuidado à } \\
\text { criança na atenção básica de saúde. }\end{array}$ \\
\hline F & Crowley & 2008 & $\begin{array}{l}\text { Collaborative Childcare Health Consultation: A } \\
\text { Conceptual Model. }\end{array}$ \\
\hline G & Barimani & 2008 & $\begin{array}{l}\text { Linkage in the chain of care: a grounded theory of } \\
\text { professional cooperation between antenatal care, } \\
\text { postpartum care and child health care. }\end{array}$ \\
\hline H & Berlin & 2008 & $\begin{array}{l}\text { Primary Child Health Care Nurses' assessment of health } \\
\text { risks in children of foreign origin and their parents - a } \\
\text { theoretical model. }\end{array}$ \\
\hline I & Fagerskiold & 2006 & \begin{tabular}{l} 
Support of fathers of infants by the child health nurse. \\
\hline
\end{tabular} \\
\hline
\end{tabular}

Em todos os artigos os autores evidenciaram a atuação do profissional de saúde no atendimento as crianças na APS, como se observa no quadro 2 .

Quadro 2: Temática abordada e objetivo de pesquisa dos artigos selecionados.

\begin{tabular}{|c|c|c|}
\hline ARTIGO & TEMÁTICA ABORDADA & OBJETIVO DO ESTUDO \\
\hline$A$ & $\begin{array}{l}\text { Limitações para utilizaçãc } \\
\text { das pesquisas como fonte de } \\
\text { conhecimento nas consultas } \\
\text { de enfermagem para } \\
\text { vacinação de crianças. }\end{array}$ & $\begin{array}{l}\text { oDescrever as fontes de informação, bem } \\
\text { ecomo barreiras e facilitadores para a utilização } \\
\text { śde pesquisa durante as consultas por } \\
\text { aenfermeiros de saúde pública relacionada à } \\
\text { vacinação infantil. }\end{array}$ \\
\hline B & $\begin{array}{l}\text { Parceria entre família } \\
\text { enfermeiros em escolas } \\
\begin{array}{l}\text { primárias no cuidado de } \\
\text { saúde. }\end{array}\end{array}$ & $\begin{array}{l}\text { e Desenvolver uma teoria substantiva } \\
\text { enrientadora de cooperação entre enfermeiros } \\
\text { de escolas primárias e famílias. }\end{array}$ \\
\hline
\end{tabular}




\begin{tabular}{|c|c|c|}
\hline C & $\begin{array}{l}\text { Buscar o entendimento do } \\
\text { cuidado à criança de várias } \\
\text { perspectivas: enfermeiros, } \\
\text { mães, gestores e docentes. }\end{array}$ & $\begin{array}{l}\text { Compreender modos de cuidar e de cuidado à } \\
\text { criança na Atenção Primária de Saúde. }\end{array}$ \\
\hline$D$ & $\begin{array}{l}\text { Cuidados paliativos à criança } \\
\text { com câncer em casa. }\end{array}$ & $\begin{array}{l}\text { Explorar experiências de profissionais de } \\
\text { cuidado primário e o seu envolvimento em } \\
\text { cuidados paliativos a crianças com câncer em } \\
\text { casa. }\end{array}$ \\
\hline$E$ & $\begin{array}{l}\text { Integração do cuidado entre } \\
\text { família, comunidade e equipe } \\
\text { de saúde. }\end{array}$ & $\begin{array}{l}\text { Compreender os fatores limitadores para a } \\
\text { integralidade do cuidado à criança na atenção } \\
\text { básica. }\end{array}$ \\
\hline$F$ & $\begin{array}{l}\text { Relacionamento } r \\
\text { diretores de centros de } \\
\text { cuidado a crianças e os } \\
\text { consultores de enfermagem. }\end{array}$ & $\begin{array}{l}\text { Explorar a natureza de consulta entre os } \\
\text { diretores de centro de acolhimento de } \\
\text { crianças e consultores de saúde e identificar } \\
\text { os fatores que promovem ou inibem uma } \\
\text { relação de colaboração. }\end{array}$ \\
\hline$G$ & $\begin{array}{l}\text { Cooperação profissional entre } \\
\text { o pré-natal, pós-parto e } \\
\text { cuidados de saúde infantil. }\end{array}$ & $\begin{array}{l}\text { Explorar a relação de coooperação entre } \\
\text { profissionais de saúde na cadeia de cuidado } \\
\text { entre pré-natal, pós-parto e cuidados de } \\
\text { saúde da criança de novos pais. Além de } \\
\text { conceitualizar barreiras e facilitadores desta } \\
\text { cooperação para gerar um modelo teórico a } \\
\text { partir das experiências dos profissionais de } \\
\text { saúde. }\end{array}$ \\
\hline $\mathrm{H}$ & $\begin{array}{l}\text { Dificuldades de interação } \\
\text { (cuidado de alta qualidade e } \\
\text { igualitário) entre enfermeiros } \\
\text { da atenção primária a saúde } \\
\text { da criança e familiares de } \\
\text { estrangeiros. }\end{array}$ & $\begin{array}{l}\text { Investigar a fundo os problemas vivenciados } \\
\text { pelos enfermeiros da atenção primária de } \\
\text { saúde na sua interação com as crianças e } \\
\text { pais de origem estrangeira. }\end{array}$ \\
\hline I & $\begin{array}{l}\text { Apoio por parte } \text { de } \\
\text { enfermeiros de saúde da } \\
\text { criança aos pais de recém- } \\
\text { nascidos }\end{array}$ & $\begin{array}{l}\text { Identificar as expectativas dos pais de } \\
\text { crianças sobre o cuidado de saúde infantil } \\
\text { incluindo os enfermeiros. }\end{array}$ \\
\hline
\end{tabular}

Em se tratando de pesquisas qualitativas, utilizando a TFD como abordagem metodológica, esperava-se que a coleta e análise de dados tivessem partido de uma questão norteadora. Contudo, apenas cinco artigos descreveram explicitamente, sendo estes o artigo B, C, E, G e I. Para esclarecer o percurso metodológico dos artigos selecionados foram sintetizadas no quadro 3 as informações consideradas mais relevantes. 
Quadro 3: Síntese dos métodos dos artigos selecionados.

\begin{tabular}{|c|c|c|c|c|}
\hline ARTIGO & AMOSTRA & $\begin{array}{l}\text { GRUPOS } \\
\text { AMOSTRAIS }\end{array}$ & \begin{tabular}{|l} 
PERÍODO \\
DE \\
COLETA
\end{tabular} & $\begin{array}{c}\text { FUNDAMENTAÇÃO } \\
\text { TFD }\end{array}$ \\
\hline A & 16 enfermeiros de saúde pública. & Não & \begin{tabular}{|c|} 
Não \\
informou
\end{tabular} & Charmaz \\
\hline B & $\begin{array}{l}22 \text { crianças de escolas primárias, } \\
19 \text { pais de alunos e } 20 \text { enfermeiros } \\
\text { das escolas primárias. }\end{array}$ & Não & $\begin{array}{c}\text { Não } \\
\text { informou }\end{array}$ & Strauss \\
\hline C & $\begin{array}{l}29 \text { gestores de saúde, profissionais } \\
\text { de saúde da UBS, médicos da } \\
\text { ESF, coordenadores de cursos de } \\
\text { graduação e pós-graguação e } \\
\text { mães. }\end{array}$ & $\begin{array}{c}\text { Sim }(5 \\
\text { grupos })\end{array}$ & 7 meses & Não informou \\
\hline D & $\begin{array}{lll}10 \text { Médicos clínicos gerais, } & 37 \\
\text { enfermeiros comunitários e } & 12 \\
\text { crianças em cuidados paliativos. }\end{array}$ & Não & \begin{tabular}{|c|} 
Até 3 \\
meses \\
após a \\
morte das \\
crianças
\end{tabular} & Não informou \\
\hline $\mathrm{E}$ & $\begin{array}{l}29 \text { gestores de saúde, profissionais } \\
\text { de saúde da UBS, médicos da } \\
\text { ESF, coordenadores de cursos de } \\
\text { graduação e pós-graduação e } \\
\text { mães. }\end{array}$ & $\begin{array}{c}\operatorname{Sim}(5 \\
\text { grupos })\end{array}$ & 7 meses & Não informou \\
\hline $\mathrm{F}$ & \begin{tabular}{|lcc}
10 pares de diretores & de \\
acolhimento de crianças & e \\
consultores de enfermagem. & \\
\end{tabular} & Não & $\begin{array}{l}9 \text { a } 12 \\
\text { meses }\end{array}$ & Charmaz \\
\hline G & rteiras & $\begin{array}{l}\text { Sim }(5 \\
\text { grupos) }\end{array}$ & 7 meses & $\begin{array}{l}\text { Strauss e } \\
\text { Charmaz }\end{array}$ \\
\hline $\mathrm{H}$ & $\begin{array}{l}15 \text { enfermeiras da atenção primária } \\
\text { de saúde da criança. }\end{array}$ & Não & \begin{tabular}{c|} 
Não \\
informou
\end{tabular} & $\begin{array}{l}\text { Glasser and } \\
\text { Strauss }\end{array}$ \\
\hline I & $\begin{array}{l}20 \text { pais de crianças menores de } \\
\text { seis meses. }\end{array}$ & Não & 13 meses & Não informou \\
\hline
\end{tabular}

Ainda sobre o percurso metodológico, em quatro artigos a teoria de base foi o interacionismo simbólico (artigos $\mathrm{F}, \mathrm{G}, \mathrm{H} \mathrm{e} \mathrm{I}$ ) e os demais não relataram uma teoria. A maioria dos artigos afirmou ter realizado comparação constante, com exceção do artigo $G$ que não mencionou na descrição do método ter utilizado esse processo para análise dos dados. A amostragem teórica foi realizada por todos os artigos, como parte do método TFD. Apesar de cinco artigos construírem um modelo teórico (artigos A, B, F, G e H) para orientar a prática, apenas o artigo H o validou. Para a etapa de coleta de dados os autores utilizaram estratégias semelhantes, tais como: 
entrevistas individuais, entrevista em grupo, grupos focais, discussões de casos, notas de campo, questionários e memorandos. Contudo foi possível observar que nem todos mencionaram fazer uso dos memorandos, estando esse presente apenas em 3 artigos ( $\mathrm{A}, \mathrm{H}$ e I).

No que se referem aos resultados dos artigos, tendo em vista que os artigos selecionados cumpriram os critérios de seleção do presente estudo, e utilizaram como método a TFD, todos desenvolveram categorias e subcategorias. Sendo que a maioria apresentou uma categoria central, com exceção do artigo D. No quadro 4 estão sintetizados os principais resultados dos artigos selecionados: produto gerado para auxiliar a atenção à criança, impacto para a prática e os avanços que a pesquisa trouxe.

Quadro 4: Síntese dos resultados dos artigos selecionados.

\begin{tabular}{|c|c|c|c|}
\hline ARTIGO & PRODUTO & IMPACTO & AVANÇOS \\
\hline$A$ & $\begin{array}{l}\text { Desenvolvimento de um } \\
\text { modelo teórico que auxiliou na } \\
\text { identificação das facilidades er } \\
\text { dificuldades dos enfermeiros } \\
\text { em utilizar pesquisas recentes } \\
\text { para prestar informações sobre } \\
\text { vacinas para os pais das } \\
\text { crianças. }\end{array}$ & $\begin{array}{l}\text { As enfermeiras poderão } \\
\text { prestar informações mais } \\
\text { atuais se vencerem as } \\
\text { barreiras encontradas e } \\
\text { utilizar os fatores facilitadores } \\
\text { a seu favor. }\end{array}$ & $\begin{array}{l}\text { Modelo teórico para auxiliar a } \\
\text { senfermagem a prestar } \\
\text { einformação esclarecida e atual } \\
\text { s sobre vacinação. }\end{array}$ \\
\hline B & $\begin{array}{l}\text { Teoria desenvolvida que pode } \\
\text { ser utilizada em um contexto } \\
\text { internacional para melhoria da } \\
\text { atenção à saúde da criança, } \\
\text { uma vez que busca melhorias } \\
\text { na cooperação entre crianças, } \\
\text { suas famílias e os enfermeiros } \\
\text { das escolas primárias. }\end{array}$ & $\begin{array}{l}\text { Ajuda a melhorar } \\
\text { compreensão da estrutura e } \\
\text { da diversidade da cooperação } \\
\text { entre a família e a enfermeira } \\
\text { da escola primária }\end{array}$ & $\begin{array}{l}\text { Gerou e complementou } \\
\text { informações existentes sobre } \\
\text { a cooperação entre famílias } \\
\text { nos serviços de saúde das } \\
\text { escolas primárias; os sujeitos } \\
\text { precisam estar cientes do seu } \\
\text { papel, a enfermagem e os } \\
\text { pais devem ter um canal de } \\
\text { comunicação para a obtenção } \\
\text { de resultados satisfatórios. }\end{array}$ \\
\hline$C$ & $\begin{array}{l}\text { Base para que os profissionais } \\
\text { de saúde e enfermeiros } \\
\text { reconheçam que cuidar tem } \\
\text { sentidos } \\
\text { fundamentados no valor da } \\
\text { vida e na dignidade humana. }\end{array}$ & $\begin{array}{l}\text { Amplia a } \\
\text { do cuida } \\
\text { criança n }\end{array}$ & $\begin{array}{lrr}\text { Ampliação do } & \text { sentido do } \\
\text { aldar em } & \text { diferentes } \\
\text { perspectivas } & \\
\text { particularidades. } & \end{array}$ \\
\hline D & $\begin{array}{l}\text { O trabalho conjunto de médicos } \\
\text { e enfermeiros em cuidados } \\
\text { paliativos a crianças com } \\
\text { câncer auxilia na morte pacifica } \\
\text { e saúde pública relacionada à } \\
\text { vacinação infantil. }\end{array}$ & $\begin{array}{l}\text { Destaca a atuação da equipe } \\
\text { mutliprofissional em cuidados } \\
\text { paliativos a família de criança } \\
\text { com câncer e importância } \\
\text { dessa prática. }\end{array}$ & $\begin{array}{l}\text { Permitiu que os profissionais } \\
\text { s de saúde da APS ganhassem } \\
\text { acesso a uma área incomum } \\
\text { acomo de cuidados paliativos } \\
\text { às crianças com câncer }\end{array}$ \\
\hline
\end{tabular}




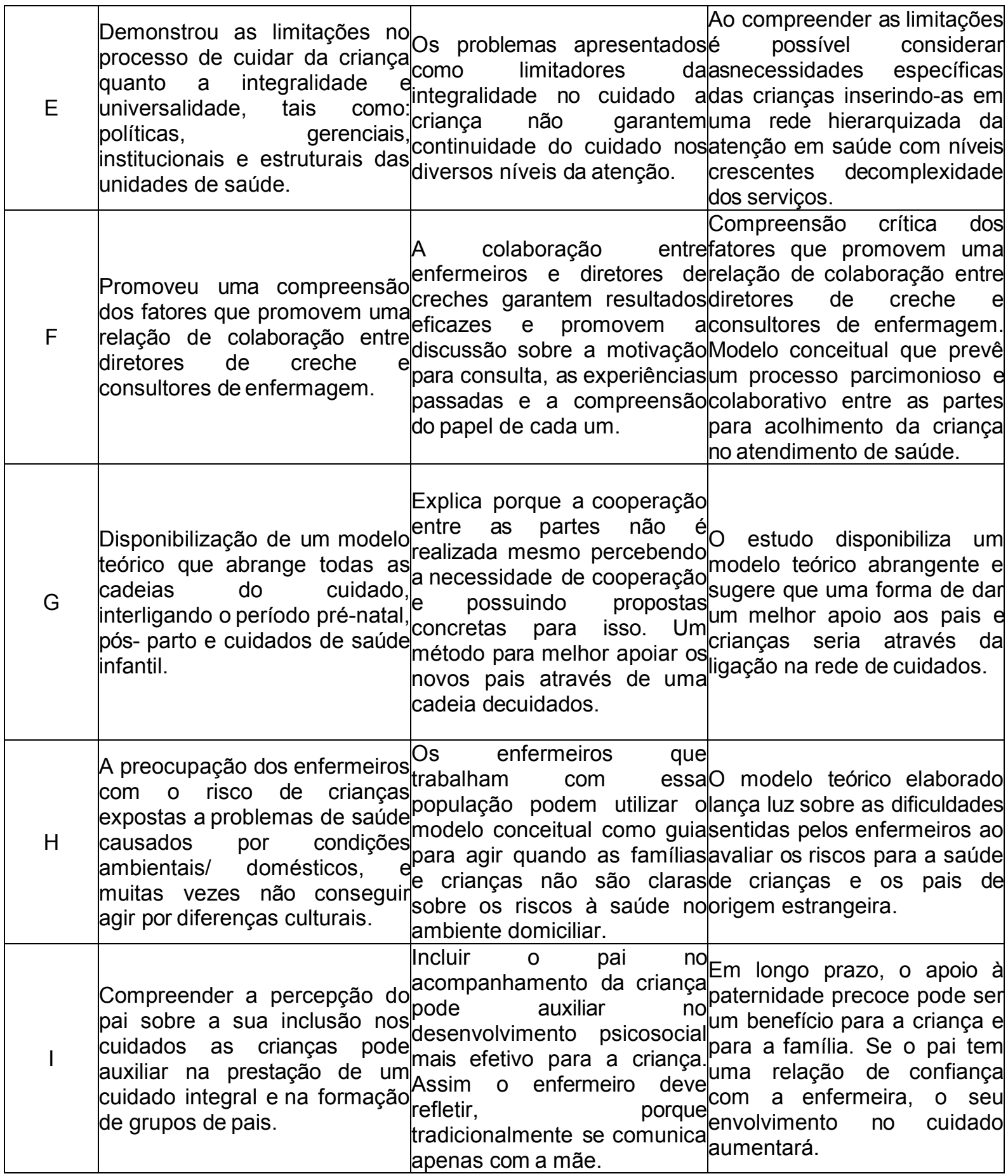

Por fim, destaca-se que diante dos resultados e conclusões apresentados pelos nove artigos selecionados, considerou-se que todos são passíveis de serem implementados na prática. 


\section{DISCUSSÃO}

A análise dos artigos que integram essa revisão revela que a metodologia TFD é amplamente utilizada, internacionalmente, pelos profissionais de saúde. Foi possivel perceber que os profissionais de enfermagem pediátrica demonstram interesse no desenvolvimento de pesquisas que adotam a TFD como método, mesmo abordando temáticas diversificadas. Esse é um fato engrandecedor para a profissão, visto que a elaboração de teorias de médio alcance contribui para o avanço da disciplina de enfermagem (FAWCETT, 2005) e a TFD pode estabelecer modelos teóricos ou reflexões teóricas como um dos produtos da aplicação deste método (DANTAS et al, 2009).

A maior parte dos artigos encontrados busca a percepção dos enfermeiros sobre a interação com a família das crianças receptoras dos cuidados (A, B, D, E, G, He I). Contudo, também é possível perceber análise de outros tipos de investigações sobre relações com outros profissionais de saúde (C, E e F). A Teoria Fundamentada em Dados (TFD) é uma metodologia própria para esses tipos de pesquisas qualitativas, pois busca compreender o significado das relações e interações entre os fenômenos sociais, o entendimento da realidade, bem como da vida e da ação humana no mundo real (BACKS et. al., 2011). Isso pode ser observado em todos os objetivos propostos pelos artigos selecionados (Quadro 2).

Quando se trata da questão de pesquisa, é importante destacar que, por se tratar de um método indutivo, onde as hipóteses serão geradas durante a análise dos dados coletados, torna-se importante a escolha do problema e a declaração da questão de pesquisa no início do processo. Essa leva o pesquisador a examinar os dados de uma maneira específica e a escolher as técnicas de coleta e análise dos dados; dá o tom para o projeto de pesquisa e ajuda o pesquisador a se manter focado, mesmo quando há massas de dados. Inicialmente, a questão é bastante ampla e aberta, mas tende a ser refinada durante a pesquisa (STRAUSS, 2009).

Analisando o percurso metodológico dos artigos encontrados, percebeu-se que, dos artigos selecionados, três trabalharam com grupos amostrais (Quadro 3). 
Esses grupos podem surgir na necessidade de alcançar o objetivo e responder à questão de pesquisa. À medida que os dados são coletados, esses são submetidos à análise concomitante, visando a saturação teórica, a qual possibilita a emersão de possíveis grupos amostrais (DANTAS, 2009).

Alguns artigos não descreveram o tempo de coleta dos dados; contudo, o intervalo de tempo determinado pelos que apresentaram em suas pesquisas foi de 7 a 13 meses. Esse intervalo é determinado pela saturação teórica. Por saturação teórica, entende-se o momento em que nenhuma nova informação surge no processo de codificação ou o novo coletado não adiciona mais explicação e não produz para a pesquisa (STRAUSS, 2009).

Entendendo que a TFD possui duas principais linhas de pensamentos (positivista - Strauss e Corbin e construtivista - Charmaz), 4 dos artigos selecionados ( $A, B, F$ e H) adotaram uma dessas linhas e descreveram em seus métodos e um deles (G) adotou parte da metodologia de Satrauss e Corbin e parte da Charmaz. Dos que identificaram, 2 ( $\mathrm{B} \mathrm{e} \mathrm{H}$ ) decidiram adotar o modelo mais conservador $\mathrm{e}$ estruturado; pode-se dizer que Glaser e seus seguidores (Strauss e Corbin) atribuiram à Teoria Fundamentada um empirismo controlado. (CHARMAZ, 2009). Já os que adotaram as teorias de Charmaz (A e F) o fizeram por entender que os pesquisadores devem se impor com postura crítica e ganhar caminho em sua narrativa (CHARMAZ, 2009). O método misto, adotado pelo artigo G, permite traçar o caminho da análise metodologicamente mais estruturada e narrativamente mais aberta (Quadro 3).

O embasamento teórico/filosófico é um importante aspecto do método (TFD). O Interacionismo Simbólico foi adotado de forma predominante pelos artigos selecionados nesta revisão. Trata-se de uma perspectiva teórica que compreende que a sociedade é construída por meio da interação, através da linguagem e comunicação. Essa foi a corrente adotada por Strauss e por isso é mais comumente utilizadas nas pesquisas com TFD (CHARMAZ, 2009). Contudo, outras correntes filosóficas podem ser utilizadas, já que à medida que a metodologia permite uma fundamentação pragmática nos dados, também possibilita a análise interpretativa (CHARMAZ, 2009). 
Os métodos de comparação constante e amostragem teórica são intrínsecos à metodologia analisada. Por esse motivo, foram adotados por todos os artigos analisados. No processo de comparação constante, todos os dados são delimitados de acordo com o princípio da saturação teórica, ao passo que as informações são coletadas, codificadas e analisadas, a fim de apontar os eventos por categorias. Assim, a coleta é efetuada até acontecer a saturação teórica, a repetição ou ausência de dados (DANTAS et al, 2009).

Alguns dos artigos analisados desenvolveram apenas as categorias e subcategorias sem, contudo, desenvolver um modelo teórico para suporte da prática de enfermagem com crianças na APS. Apesar de a TFD possibilitar a construção de teoria com base nos dados investigados de determinada realidade, também é possível estabelecer modelos teóricos ou reflexões teóricas (DANTAS et al, 2009). Dos que construíram modelos teóricos, apenas um validou o mesmo. O processo de validação permitiu ao pesquisador desenvolver um modelo teórico que pode ser considerado como consistente e clinicamente relevante (BERLIN, 2008).

As estratégias de coleta de dados foram diversificadas, desde grupos focais a entrevistas individualizadas. Para a elaboração de uma teoria sólida, a coleta de dados precisa ser relevante e fornecer material para análise significativa. Para isso, os dados precisam ser detalhados, completos e focados. Isso implica em extensivas notas de campo de observação, coleta de relatos pessoais por escrito e reunião de narrativas detalhadas (CHARMAZ, 2009). A ausência de memorandos por algumas das pesquisas foi destaque, visto que estes consistem em uma forma de registro referente à formulação de teorias (DANTAS et al, 2009) e são uma forma eficaz de desmembrar e analisar os códigos gerados a partir dos dados (CHARMAZ, 2009).

A codificação é onde serão gerados os ossos que definirão a estrutura esquelética (analítica) a partir da qual se constrói a análise. Manifestam-se como elementos da teoria nascente que explica os dados e orientam novas coletas, bem como novas questões de pesquisa. (CHARMAZ, 2009).

O produto gerado pelos estudos selecionados demonstrou a disponibilidade científica de uma teoria (base teórica) ou um modelo teórico que sustentasse a prática de atenção aos familiares, aprimorasse a relação entre a equipe de saúde ou 
aperfeiçoasse o cuidado de enfermagem diretamente as crianças da APS. Teorizar não significa apenas elaborar conceitos, mas formular em um esquema lógico, sistemático e explanatório que relacione os conceitos e não apenas organize-os (STRAUSS, 2009).

O objetivo da teorização é desenvolver teorias úteis (STRAUSS, 2009). Por esse motivo, é importante perceber que as pesquisas aqui estudadas originaram impactos e avanços no conhecimento da enfermagem com a construção de teorias para que a enfermagem possa: prestar uma assistência atual e esclarecida, cooperar com outros serviços de saúde e educação, ampliar o entendimento de um cuidar integral, adentrar em ambientes poucos explorados como o dos cuidados paliativos e desenvolver relação satisfatória com os familiares.

Dessa forma, percebeu-se que os estudos desenvolvidos para assistência de enfermagem a crianças na APS utilizando a TFD como método de pesquisa, em sua maior parte, cumpriram os critérios de uma teoria fundamentada completa, que deve se ajustar aos dados, ter utilidade, densidade conceitual, durabilidade, passível de alteração e possuir poder explicativo (CHARMAZ, 2009). A partir disto, podem alcançar os objetivos de ser passível de aplicação da prática, de fácil entendimento e utilização e aplicável a todas as populações.

\section{CONCLUSÃO}

A partir desta revisão integrativa, foi possível determinar que a TFD tem sido utilizada para pesquisas realizadas pelos profissionais de enfermagem, o que pode auxiliar no desenvolvimento teórico e científico da profissão. Os principais objetivos das pesquisas desenvolvidas com crianças na APS, que utilizam TFD como metodologia, estiveram direcionadas para a relação família/comunidade e enfermeiro, enfermeiro/criança e enfermeiro e demais profissionais da saúde.

A questão de pesquisa não foi mostrada de forma explícita em alguns dos artigos, o que poderia prejudicar a formulação da teoria; contudo, a maior parte dos artigos seguiu o percurso metodológico previsto ao utilizarem os princípios de 
saturação teórica, amostragem teórica e comparação constante. Os autores se mostraram mais positivistas quando adotaram a linha de pensamento de Strauss e Corbin e o interacionismo simbólico como base teórico/filosófico; entretanto, é possível perceber um avanço na linha de pensamento construtivista, não sendo possível medir o impacto deste nesse estudo.

O desenvolvimento de modelos teóricos está em crescimento, contudo a sua validação ainda é baixa quando se sabe da credibilidade e relevância para a prática clínica de enfermagem pediátrica. Além disso, as pesquisas têm originado produtos consistentes, que gerarão impactos e avanços para a área, de forma a prestar um cuidado integral, com vínculo e atual.

Esta revisão integrativa é importante por sumarizar e integrar o conhecimento que tem sido gerado para os profissionais de enfermagem pediátrica da APS através da TFD. Porém, apresenta como limitação os critérios de seleção dos artigos, ao incluir somente os cuidados de enfermagem, impossibilitou conhecer quais profissionais da saúde mais têm utilizado a TFD como método.

\section{REFERÊNCIAS BIBLIOGRÁFICAS}

WORLD HEALTH ORGANIZATION. Atención primaria de salud. Informe de la Conferencia Internacional sobre Atención Primaria de Salud. Alma-Ata, URSS, 6-12 de septiembre de 1978. Geneva: WHO, 1978.

PAN AMERICAN HEALTH ORGANIZATION (PAHO). Renewing Primary Health Care in the Americas, 2007 Edition ISBN 9275126984.

SOUSA, Francisca Georgina Macedo de; ERDMANN, Alacoque Lorenzini; MOCHEL, Elba Gomide. Modelando a integralidade do cuidado à criança na Atenção Básica de Saúde. Rev Gaúcha Enferm., Porto Alegre (RS) 2010dez;31(4):701-7. Disponível em: https://seer.ufrgs.br/index.php/RevistaGauchadeEnfermagem/article/view/12974/11852 Acesso em: 18 de Fev de 2020.

SOUZA, Macela Tavares; SILVA, Michelly Dias da; CARVALHO, Rachel. Revisão integrativa: o que é e como fazer. Einstein (São Paulo) [internet]. 2010 [citado 2014 jan. 5]; 8(1):102-6. Disponível em: http://astresmetodologias.com/material/O_que_e_RIL.pdf. Acesso em: 18 de Fev de 2020.

GASTALDON, B.; MARTINS, J.C.; POLTRONIÉRI, K.V. Obesidade infantil: um problema do presente com olhares para o futuro - promovendo o ser e a família saudável no quotidiano junto à enfermagem. Trabalho de Conclusão de Curso - Universidade Federal de Santa Catarina, $\quad$ Florianópolis, 2007.2 Disponível em: http://www.bibliomed.ccs.ufsc.br/ENF0468.pdf. Acesso 17 de Nov. 2011 
SARTI, Thiago Dias et al . Avaliação das ações de planejamento em saúde empreendidas por equipes de saúde da família. Cad. Saúde Pública, Rio de Janeiro, v. 28, n. 3, Mar. 2012. Available from <http://www.scielosp.org/scielo.php?script=sci_arttext\&pid=S0102311X2012000300014\&lng=en\&nrm=iso>. access on $02-$ Oct. 2012. http://dx.doi.org/10.1590/S0102-311X2012000300014.

FAWCETT, Jaqueline. Middle range theories are necessary for the advancement of the $\begin{array}{lllll}\text { discipline. } & \text { Aquichan } & \text { [s.d.p.]. } & 2005 & \text { Disponivel } \\ \text { em: }\end{array}$ http://aquichan.unisabana.edu.co/index.php/aquichan/article/view/57/119 Acess on 07 Apr. 2014.

DANTAS, Claudia de Carvalho et al . Teoria fundamentada nos dados - aspectos conceituais e operacionais: metodologia possível de ser aplicada na pesquisa em enfermagem. Rev. Latino-Am. Enfermagem, Ribeirão Preto, v. 7, n. 4, Aug. 2009. Available from $<$ http://www.scielo.br/scielo.php?script=sci_arttext\&pid=S010411692009000400021\&lng=en\&nrm=iso $>$. access on 26 Sept. 2012. http://dx.doi.org/10.1590/S0104-11692009000400021.

BACKES, Marli Terezinha Stein et al. Desenvolvimento e validação de teoria fundamentada em dados sobre o ambiente de unidade de terapia intensiva. Esc. Anna Nery, Rio de Janeiro, v. 15, n. 4, Dec. 2011. Available from $<$ http://www.scielo.br/scielo.php?script=sci_arttext\&pid=S1414-

81452011000400016\&lng=en\&nrm=iso $>$. access on 10 Oct. 2012. http://dx.doi.org/10.1590/S1414-81452011000400016.

MENDES, Karina Dal Sasso; SILVEIRA, Renata Cristina de Campos Pereira; GALVAO, Cristina Maria. Revisão integrativa: método de pesquisa para a incorporação de evidências na saúde e na enfermagem. Texto contexto - enferm., Florianópolis , v. 17, n. 4, p. 758-764, Dec. 2008 . Available from <http://www.scielo.br/scielo.php?script=sci_arttext\&pid=S010407072008000400018\&lng=en\&nrm=iso>. access on 18 Feb. 2020. https://doi.org/10.1590/S0104-07072008000400018.

WORLD HEALTH ORGANIZATION. Social determinants of heal-th and well-being among young people: Health Behaviour in School-Aged Children (HBSC) study: international report from the 2009/2010 survey [Internet]. Copenhagen WHO Regional Office for Europe; 2012 [cited 2014 Jan 5]. Avai-lable from: http://www.euro.who.int/ data/assets/pdf_fi- le/0003/163857/Socialdeterminants-of-health-and-well-being-among-young-people.pdf.

FAWCETT J. Contemporary nursing knowledge: analysis and evaluation of nursing models and theories. 2nd ed. Philadelphia: F. A. Davis; 2005.

STRAUSS, A; CORBIN, J. Pesquisa Qualitativa. Técnicas e procedimentos para o desenvolvimento de Teoria Fundamentada. 2. ed. Porto Alegre: Artmed, 2009. 288p.

CHARMAZ, K. A construção da teoria fundamentada: guia prático para a análise qualitativa, tradução Joice Elias Costa. Porto Alegre: Artmed, 2009.

BERLIN A, HYLANDER I, TÖRNKVIST L. Primary Child Health Care Nurses' assessment of health risks in children of foreign origin and their parents a theoretical model. Scand $\mathrm{J}$ Caring Sci. 2008 Mar;22(1):118-27. doi: 10.1111/j.1471-6712.2007.00533.x. Acesso em 15/01/2015. Disponível em: http://www.ncbi.nlm.nih.gov/pubmed/18269431 CASP, Critical Appraisal Skills Programme (CASP) Qualitative Research Checklist, 2013. Disponí vel em: http://media.wix.com/ugd/dded87_951541699e9edc71ce66c9bac4734c69.pdf. 